Published in final edited form as:

Nat Cell Biol. 2013 January ; 15(1): 13-15. doi:10.1038/ncb2665.

\title{
Humans put their eggs in more than one basket
}

\author{
Diana J. Laird \\ Department of Ob/Gyn, Center for Reproductive Sciences, Eli and Edythe Broad Center for \\ Regeneration Medicine and Stem Cell Science, UCSF, 35 Medical Center Way, San Francisco, \\ CA USA 94143
}

\section{Abstract}

Primordial germ cell (PGC) development in the human fetus remains relatively uncharted. A new study suggests that epigenetic reprogramming and sex differentiation in human PGCs occur asynchronously over an extended time period. This raises new questions and implications for in vitro PGC differentiation.

\begin{abstract}
Humans reproduce in the second through fourth decade of life, and yet the gametic precursors arise in the first weeks of embryogenesis. By the time pregnancy is detected, the cells in our children destined to beget our grandchildren have already been established, expanded, mobilized, and are on the cusp of their first act of rebellion: erasing genomic imprints of their parents. Our germline, the cell lineage committed to the exclusive generation of gametes is sequestered from somatic lineages for most of development. In mammals, soma-germline divergence occurs during gastrulation, with the allocation of primordial germ cells (PGCs) from the epiblast. PGCs then traverse the embryo over the ensuing days before reaching the gonads. Only then sex-specific differentiation begins, with meiosis in female and mitotic arrest in male ${ }^{1}$. Our knowledge of mammalian germ cell development comes primarily from the study of mice, and so far, precious little is known about germ cells in human development. On page XX of this issue ${ }^{2}$, Amander Clark and colleagues expand this knowledge in a comprehensive description of human PGCs. They use sophisticated approaches to characterize one particular population of PGCs defined by the expression of cKIT, by analyzing an extensive number of fetal gonads from gestational week 6.5 through 22. In an important step for the field, their approach of flow-cytometric purification of cKIT+ cells from human fetal gonads creates the potential for unprecedented global analyses. This will inform models of ovarian reserve formation as well as analysis of in vitro gametogenesis.
\end{abstract}

Although the mode of PGC specification and PGC migratory routes have changed through evolution, their function as gametic precursors has not. Studies in model organisms have uncovered highly conserved germ cell genes, such as VASA. In mammals, pluripotency factors such as OCT4 are critical for PGC survival. Other non-specialized genes such as cKIT, which directs proliferation, survival and migration of mouse PGCs as well as neural crest and blood lineages, were most likely coopted by the germline. ${ }^{1,3}$ During first trimester human development, Gkountela et al. ${ }^{2}$ observed coexpression of many germ cell markers in the testes and ovaries, suggesting strong parallels to PGC development in mice, in which male and female germs cells initially exhibit similar expression programmes. Importantly, they confirm by single-cell PCR that the $\mathrm{cKIT}^{+}$cells purified from first trimester gonads express the full complement of known early PGC markers. However, after 12 weeks, human PGCs appear to separate into two populations of cKIT/OCT4 ${ }^{+}$and $\mathrm{VASA}^{+}$cells (Figure 1). In ovaries, the appearance of the synaptonemal complex in $\mathrm{VASA}^{+}$cells after 14 weeks indicates their meiotic entry. Divergence of human PGC gene expression and distribution was described previously, with $\mathrm{VASA}^{+}$early meiotic cells located toward the center of the ovary and cells co-expressing $\mathrm{OCT}_{4}{ }^{+}$and other germ cell markers at the periphery ${ }^{45}$. In 
contrast to the anterioposterior wave of meiosis initiation observed in fetal mouse ovaries ${ }^{6}$, the radial gradient in human ovaries suggests the existence of species differences in initiating cues, with local somatic cell interactions operating in humans versus diffusible signals in mice. The authors propose that these $\mathrm{cKIT}^{+} / \mathrm{OCT}^{+}$cells persisting in second trimester gonads represent a more primitive PGC than the $\mathrm{VASA}^{+}$cells-an idea supported by their maintenance of a core germ cell gene expression signature at the single cell level. Will these $\mathrm{KIT}^{+}$cells be culled in one of the waves of apoptosis or, as their transcriptome suggests, will they enter meiosis later? This lineage divergence may reflect establishment of an oogenesis "production line", as proposed 45 years ago ${ }^{7}$, for which first-in-first-out would be the $\mathrm{VASA}^{+}$cells whereas the laggards in meiotic entry and ovulation would be the cKIT $^{+}$ cells. Further investigation of the segregation of $\mathrm{VASA}^{+}$and cKIT ${ }^{+}$PGCs in the fetal ovary could help elucidating the basis for the rise in oocyte chromosomal abnormalities associated with increasing maternal age ${ }^{7}$. However, the functional significance of the observed PGC dichotomy in the fetal testes, where meiosis is still years away, remains to be determined.

Generation of a functional germline also requires a process that is only beginning to be understood in mice, the resetting of epigenetic marks. Chromatin modifications in mouse PGCs include depletion of the histone variant H2A.Z as well as loss of the repressive histone H3K27 trimethylation mark (H3K27me3), which occur at E10.5-11 ${ }^{8}$. Gkountela et al. show that similar chromatin modifications occur in human PGCs after 9 weeks of development. Possibly corroborating the common origin of the cKIT ${ }^{+}$and $\mathrm{VASA}^{+}$cells, they both maintained low levels of H3K27me3 and H2A.Z. Epigenetic reprogramming in mouse PGCs also involves genome-wide DNA demethylation as well as demethylation at the promoters of paternally and maternally-imprinted genes ${ }^{9}$. By analysing differentially methylated regions in the $\mathrm{cKIT}^{+}$population, the authors reveal a progressive and locusspecific demethylation at the second trimester, that echoes the dynamics in mice, although occurring relatively later. Finally, by immunostaining, reduced levels of 5-methyl cytosine and its derivative 5-hydroxymethyl cytosine were observed in human PGCs compared to surrounding somatic cells at all fetal ages obtained. This result argues that large-scale demethylation in human PGCs occurs before 7 weeks of development, the earliest time point examined. Detection of enzymes involved in DNA demethylation (TET1-3, AID and $\mathrm{TDG}^{10}$ ) in the $\mathrm{CKIT}^{+}$population is consistent with their involvement in this process. The analysis of DNA demethylation and histone modification dynamics hints that these events may occur separately in human germ cell development while being coincident in mouse PGCs. Based on their observations, the authors proposed a model of two separate periods of epigenetic reprogramming in human PGCs: an early genome-wide DNA demethylation phase occurring before 7 weeks and and a second phase, involving changes in chromatin modifications and imprint erasure in the gonadal PGCs after 9 weeks. With the current progress on methods for epigenetics analysis, study of these reprogramming events on a genome-wide scale will soon be obtainable in human PGCs.

Although this study expands knowledge of human PGC development in the fetal gonads, examination of earlier stages of development is precluded by the inaccessibility of tissues before 7 weeks. To address this gap, Gkountela et al. examined candidate PGCs differentiated from human embryonic stem cells (or esPGCs). Overlap between markers of ES cells and early PGCs hinders the unequivocal identification of esPGCs using this method, as the cells obtained following differentiation could be residual ES cells. Whereas previous cell population level analyses report expression of PGC genes in ESC lines, single cell qRT-PCR on hESCs in this study makes an important point: germ cell genes expressed in hESCs appear non-overlapping. In light of the coexpression of core germ cell genes in first trimester human PGCs, the authors apply the criterion of co-expression in single cells towards improving the protocol for differentiation of esPGCs. What emerges from these attempts is that previously optimized embryoid body culture yields cells that have not 
undergone global DNA demethylation nor expressed the late PGC genes VASA. Much like the mouse esPGCs that reach a developmental impasse equivalent to E9.5 in mouse development ${ }^{11}$, human esPGCs are most comparable to migratory PGCs before 6 weeks. Although recent work beautifully showed that co-aggregation of mouse esPGCs with fetal ovary somatic cells eventually induces their developmental progression toward a functional gamete $^{12}$, efforts by Clark and colleagues to co-culture human esPGCs with human fetal gonad stroma were unsuccessful. Nonetheless, these studies set a new standard for validation of in vitro PGCs at a single cell level, and provide a template for future efforts to re-create soma-PGC interactions in vitro.

This work inspires questions about the lineage relationships and fates of the two distinct PGC populations identified in human fetal gonads. Given the impossibility of lineage tracing or functional studies on human gamete precursors, approaching these questions experimentally requires creativity and resourcefulness. With the authors' current foray into isolating live cells from human fetal tissues by cell surface markers, the fate and niche requirements of human PGCs may be addressable by xenotransplantation studies into mouse embryonic or postnatal gonads, and by ex vivo re-aggregation studies with somatic gonads. These endeavors will advance our understanding of how PGC development gone awry contributes to human infertility or germ cell tumors. With recent demonstrations that successful mouse spermatogenesis ${ }^{13}$ and oogenesis ${ }^{12}$ from pluripotent stem cells requires co-culture with fetal gonad and transplant in vivo, the prospect of generating human gametes in vitro for treatment of infertile patients can only proceed from a deeper understanding of the development of PGCs in the embryo.

\section{References}

1. Laird DJ, von Andrian U, Wagers AJ. Cell. 2008; 32(4):612-630. [PubMed: 18295579]

2. Gkountela S, Li Z, Vincent JJ, Zhang KX, Chen A, Pellgrini M, Clark AT. Nat. Cell Biol. 2013; $\mathrm{X}: \mathrm{XXX}-\mathrm{XXX}$.

3. Mintz B, Russell ES. J. Exp. Zool. Part A Ecol. Genet. Physiol. 1957; 134:207-237.

4. Stoop H, et al. Hum Reprod. 2005; 20(6):1466-1476. [PubMed: 15734757]

5. Childs AJ, Kinnell HL, He J, Anderson RA. Stem Cells Dev. 2012; 21(13):2343-2349. [PubMed: 22296229]

6. Bullejos M, Koopman P. Mol Reprod Dev. 2004; 68(4):422-428. [PubMed: 15236325]

7. Henderson SA, Edwards RG. Nature. 1968; 218:22-28. [PubMed: 4230650]

8. Hajkova P, et al. Nature. 2008; 452:877-881. [PubMed: 18354397]

9. Hayashi K, Surani MA. Cell Stem Cell. 2009; 4:493-498. [PubMed: 19497276]

10. Saitou M, Kagiwada S, Kurimoto K. Development. 2012; 139(1):15-31. [PubMed: 22147951]

11. Vincent JJ, et al. PLoS One. 2011; 6(12):e28960. [PubMed: 22194959]

12. Hayashi K, et al. Science. 2012 Oct 4.

13. Hayashi K, et al. Cell. 2011; 146(4):519-532. [PubMed: 21820164] 


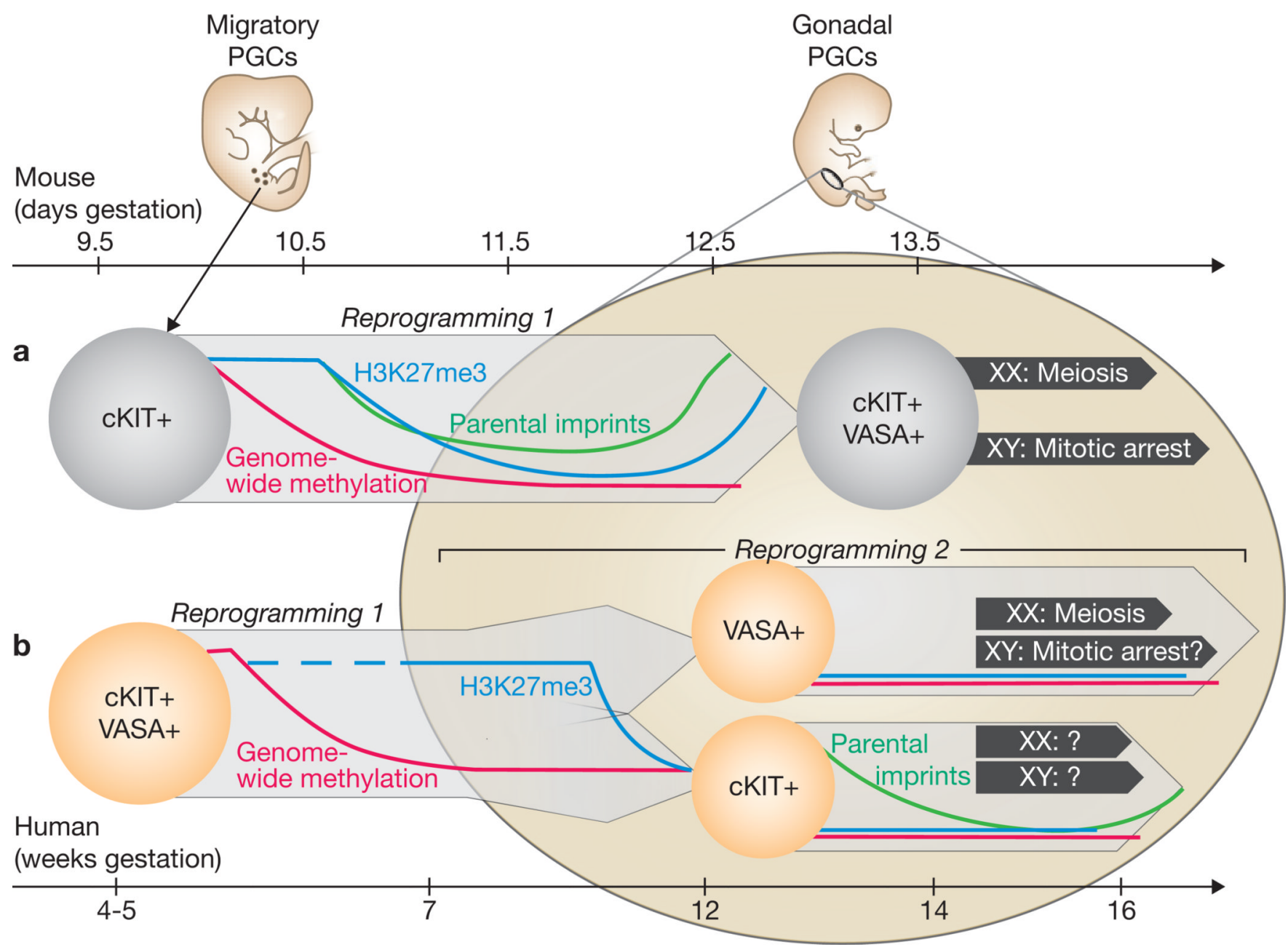

Figure 1.

Comparison of models of PGC development in mice and humans. (a) Mouse PGCs (light grey) seem to remain as a single population that upregulates late markers such as VASA upon colonizing the gonad at around stage E11. Epigenetic reprogramming in mouse PGCs occurs over approximately 3 days during late migration and early gonad colonization. (b) Human PGCs (light orange) exist as a single population during migration that bifurcates into $\mathrm{cKIT}^{+}$and $\mathrm{VASA}^{+}$lineages at 11-12 weeks, more than 1 month after reaching the gonad. Although VASA ${ }^{+}$PGCs enter meiosis in the fetal ovary, the fate of $\mathrm{cKIT}^{+} \mathrm{PGCs}$ remains unclear. Epigenetic reprogramming in human PGCs probably occurs as an early wave of genome-wide demethylation (red) and a later wave of demethylation of imprinted loci (green) and depletion of H3K27me3 (blue). 